\title{
Oral administration of the probiotic Lactobacillus fermentum VRI-003 and mucosal immunity in endurance athletes
}

\author{
A J Cox, ${ }^{1,2}$ D B Pyne, ${ }^{1,3}$ P U Saunders, ${ }^{1}$ P A Fricker ${ }^{3,4}$
}

'Department of Physiology, Australian Institute of Sport, Canberra, Australia; ${ }^{2}$ School of Biomedical Sciences, University of Newcastle, Newcastle, Australia; ${ }^{3}$ Medical School, The Australian National University, Canberra, Australia; ${ }^{4}$ Australian Institute of Sport, Canberra, Australia

\section{Correspondence to:} Dr D Pyne, Department of Physiology, Australian Institute of Sport, PO Box 176, Belconnen, ACT, 2616, Australia; david.pyne@ausport. gov.au

Accepted 21 December 2007 Published Online First 13 February 2008

\author{
ABSTRACT \\ Objective To evaluate the ability of a probiotic \\ Lactobacillus fermentum VRI-003 (PCC) to enhance the \\ mucosal immune system of elite athletes.
}

Design and setting $A$ double-blind, placebo-controlled, crossover trial was conducted over a 4-month period of winter training.

Participants 20 healthy elite male distance runners. Interventions PCC was given at a daily dose of $1.2 \times 10^{10}$ as a freeze-dried powder in gelatin capsules. Placebo capsules contained an inert excipient.

Main outcome measures Treadmill performance (monthly), mucosal and systemic immunity (monthly), training (daily) and illness (daily) were assessed. Serum cytokine levels, salivary IgA levels and incidence, duration and severity of respiratory tract infections were measured.

Results Subjects reported less than half the number of days of respiratory symptoms during PCC treatment (30 days) compared with placebo (72 days, $\mathrm{p}<0.001$ ). Illness severity was also lower for episodes occurring during the PCC treatment $(p=0.06)$. There were no significant differences in the mean change in salivary $\lg \mathrm{A}$ and IgA1 levels, or in interleukin (IL)4 and IL12 levels, between treatments. However, PCC treatment elicited a twofold ( $p=0.07$ ) greater change in whole-blood culture interferon gamma (IFN $\gamma$ ) compared with placebo. No substantial changes in running performance measures were seen over the study period.

Conclusions Prophylactic administration of PCC was associated with a substantial reduction in the number of days and severity of respiratory illness in a cohort of highly trained distance runners. Maintenance of IFN $\gamma$ levels may be one mechanism underpinning the positive clinical outcomes.

Some endurance athletes undergoing strenuous training have an increased susceptibility to upper respiratory tract infection (URTI) as a result of exercise-induced immunosuppression. ${ }^{1}$ Several studies have demonstrated that the concentration and flow rate of secretory immunoglobulin A (SIgA), the major effector of host resistance to URTI, decreases after intense endurance exercise. ${ }^{1-4}$ Gleeson and colleagues have shown a substantial correlation between a decrease in salivary IgA (particularly salivary IgA1) during training, ${ }^{5}$ and an increase in the number of respiratory infections in elite athletes. Since stress affects the levels of total salivary $\operatorname{Ig} \mathrm{A}^{6}$ measurement of salivary IgA subclasses may have value in quantifying the risk of infection in elite athletes. ${ }^{5}$ Given these observations, there is both clinical and theoretical interest in understanding the relationships between exercise, training, immune function and the practical intervention strategies to maintain immunocompetence in athletes undertaking intensive training programmes.

The efficacy of probiotics (lactic acid bacteria, which when ingested provide positive health benefits) in the treatment of gastrointestinal disease is well established. Several clinical studies have shown that certain probiotics may be useful in treating a variety of diarrhoeal disorders, including rotavirus diarrhoea, antibiotic-associated diarrhoea, Clostridium difficile diarrhoea, and traveller's diarrhoea. ${ }^{78}$ There is also evidence that probiotics stimulate mucosal immune function in addition to their primary role in intestinal health. Lactic acid bacteria are reported to have a wide range of effects on the immune system, including augmentation of phagocytic function in neutrophils, monocytes, macrophages and natural killer cells. ${ }^{9}$ Specific immune responses, both humoral and cellular, may also be enhanced by administration of lactobacilli. ${ }^{10}$ Administration of Lactobacillus to children attending day care centres elicited a moderate reduction in the number of respiratory infections. ${ }^{11}$ An initial study by our research group examined the clinical and immunological consequences of probiotic administration in habitual exercisers. These subjects were patients at a fatigue outpatient clinic rather than healthy well-performing athletes. ${ }^{12}$ Administration of the probiotic corrected a deficiency in the level of interferon $\gamma$ (IFN $\gamma)$. The only other study of probiotics in athletes reported that probiotic administration shortened the duration of gastrointestinal symptom episodes in marathon runners but had no effect on the incidence of respiratory infections or gastrointestinal symptoms. ${ }^{13}$

Another probiotic strain Lactobacillus fermentum is a potentially useful nutritional intervention in a sports medicine setting. Preliminary research in mice highlights the potential for enhancement of gut immunity with $L$ fermentum administration. ${ }^{14} L$ fermentum colonises the gastrointestinal tract of healthy volunteers after a single dose ${ }^{15}$ and adheres to Peyer's patches, the immunosampling site in the small intestine. ${ }^{16}$ We sought to extend these initial studies into healthy human subjects. The aim of this study was to determine whether $L$ fermentum enhances immunological protection in a group of healthy well-trained athletes, and whether any induced immunological changes correspond with a change in the self-reported incidence of illness and infection. We hypothesised that oral 
administration of the $L$ fermentum might trigger a protecting mucosal immune response and reduce the incidence, severity and/or duration of respiratory illness in endurance athletes.

\section{SUBJECTS AND METHODS}

\section{Study subjects and design}

Twenty highly-trained distance runners, competing in events ranging from $800 \mathrm{~m}$ to the marathon $(42.2 \mathrm{~km})$, were recruited into the 14-week study. The mean (SD) age of the study cohort at recruitment was 27.3 (6.4) years. Other characteristics (mean (SD)) of the group at recruitment were: height $1.80(0.06) \mathrm{m}$; mass 66.7 (6.0) kg; percentage muscle mass 46.6 (1.5)\%; percentage fat mass 8.1 (0.9)\%; and $\mathrm{VO}_{2 \max } 67.1$ (4.5) $\mathrm{ml} / \mathrm{kg}$ / min. The study protocol was approved by the Ethics Committee of the Australian Institute of Sport in accordance with the Code of Ethics of the World Medical Association (Declaration of Helsinki).

At recruitment a sports doctor recorded a medical history for each subject and baseline measures of immune function were taken. The study was undertaken using a double-blind, placebocontrolled, crossover design with subjects randomly assigned to placebo or treatment groups by a third party independent of the research team. The research team remained unaware of these allocations until completion of the study. After recruitment, subjects completed an initial treatment month (28 days) receiving either $L$ fermentum or placebo. A washout month (28 days) followed the completion of the first treatment month. Previous studies have established that the typical time course for passage of probiotic bacteria through the gastrointestinal tract is 3-8 days. ${ }^{17}$ Subjects were then crossed into the opposing arm of the study for a second treatment month (28 days), so that those receiving $L$ fermentum VRI-003 as the first treatment received the placebo as the second treatment and vice versa. Subjects were monitored for an additional fortnight (referred to as "follow-up") after completion of the second treatment.

During each treatment month (28 days) subjects were required to take three capsules, twice daily of either the probiotic or placebo with food. The probiotic, L fermentum VRI-003 (PCC), contained a minimum of two billion of Lactobacillus fermentum strain VRI-003 viable organisms in hard gelatin capsules with microcrystalline cellulose as the excipient. Preliminary quality assurance studies indicated a minimum of two billion viable organisms per capsule at the end of the specified shelf life. The total daily dose of six capsules provided $1.2 \times 10^{10}$ colony-forming units. The probiotic is available to healthcare practitioners as Prograstrim (Probiomics Pty Ltd, Perth, Australia). The placebo was identical to the $L$ fermentum VRI-003 except that the Lactobacillus was replaced with microcrystalline cellulose. Subjects maintained daily training and illness records, provided weekly saliva and monthly blood samples, and completed monthly treadmill testing in the laboratory. Subjects were also required to refrain from consuming yoghurt and yoghurt-based products for the duration of the study.

\section{Participant compliance}

All 20 subjects completed the study. Subjects were asked to score their compliance with the medication schedule on a fivepoint scale (1, low compliance; 5 , complete compliance). The mean levels of compliance during the first and second treatment months were 4.1 and 3.9 , respectively. The respective compliance scores were 3.9 during the $L$ fermentum VRI-003 treatment and 4.2 during the placebo treatment.

\section{Training and performance measures}

Subjects recorded details of their training during the study. For each session, training mileage $(\mathrm{km} / \mathrm{wk})$, duration $(\mathrm{h} / \mathrm{wk})$ and intensity (scored on a 1-5 scale: 1 , easy; 5 , maximal) were recorded. At the start of the study period and at the end of each study month subjects completed a treadmill running test. This test involved continuous incremental running of three 4-minute periods at 14,16 and $18 \mathrm{~km} / \mathrm{h}$ at $0 \%$ gradient. After a 2 -minute break the subject started running again at a speed of $18 \mathrm{~km} / \mathrm{h}$ that was increased by $1 \mathrm{~km} / \mathrm{h}$ each minute for 3 minutes all at $0 \%$ gradient. Beyond this point the speed was maintained at $20 \mathrm{~km} / \mathrm{h}$ but the gradient increased by $1 \%$ per minute until the subject reached volitional exhaustion. Heart rate was recorded continuously with expired ventilation samples collected for determination of oxygen uptake. Improvements or otherwise in endurance fitness were indicated by the maximal oxygen uptake and performance level (running velocity and gradient) reached at the end of the incremental treadmill test.

\section{Patterns of illness}

Subjects kept a daily symptom log to record any symptoms of illness (and side effects) including episodes of sore throat, cough, runny nose, sneezing (URTI), chest congestion (lower respiratory illness (LRI)), headache, fever, muscle or joint aches, abdominal pain, cramping, and/or loss of appetite. Subjects rated the severity of the symptoms as mild (normal training), moderate (modified training) or severe (trained discontinued) (scored 1-3, respectively). Dietary supplements and drugs (prescribed and over-the-counter) that might have influenced the underlying immune function or exercise performance, or both, were also recorded. Illness logs were reviewed by a sports doctor for coding of reported episodes of illness. At the end of each treatment subjects were asked to score their general health on a five-point scale (1, frequent illness; 5 , no illness). Where possible, acute presentation for doctor verification and documentation was undertaken.

\section{Measures of mucosal immunity}

Whole, mixed, unstimulated saliva was collected by passive drool or gentle spitting and stored at $-80^{\circ} \mathrm{C}$ until analysis for IgA, IgA1 and salivary albumin. SIgA concentrations in saliva were measured using an in-house ELISA assay as previously described..$^{5}$ All samples were assayed in triplicate in conjunction with high, moderate and low positive controls and saliva from an IgA-deficient person used as a negative control. SIgA concentrations were determined by standardisation against Human Serum Protein Calibrator (Dako, Glostrup, Denmark) referenced against BCR CRM470. The interassay variability for the high, moderate and low positive controls was $11.4 \%, 11.7 \%$ and $8.9 \%$, respectively.

\section{Measures of systemic immunity}

Upon recruitment and at the completion of treatment 1 , washout and treatment 2, subjects provided a blood sample for establishment of whole-blood cultures and determination of interleukin (IL)4 (Th2 secreted), IL12 (macrophage secreted) and IFN $\gamma$ (Th1 secreted) concentrations. ${ }^{18} 19$ Blood was collected from the antecubital vein using standard venepuncture techniques. Whole-blood cultures were established at $37^{\circ} \mathrm{C}$ and $5 \%$ $\mathrm{CO}_{2}$ for 48 hours; culture supernatants were then collected and cytokine concentrations determined using in-house ELISA methods as previously described. ${ }^{20} 21$ For the IL4 ELISA the interassay variability for high and low controls was $9.8 \%$ and 
Table 1 The training mileage, duration, intensity, $V_{0}{ }_{2 m a x}$ and maximum treadmill time recorded from the cohort during each of the treatments

\begin{tabular}{llllll}
\hline & $\begin{array}{l}\text { Mileage } \\
(\mathbf{k m} / \mathbf{w k})\end{array}$ & $\begin{array}{l}\text { Duration } \\
(\mathbf{h} / \mathbf{w k})\end{array}$ & $\begin{array}{l}\text { Intensity } \\
\text { (scored } \mathbf{1 - 5})\end{array}$ & $\begin{array}{l}\mathbf{V}_{\mathbf{0}_{\text {2max }}} \\
(\mathbf{m} / \mathbf{k g} / \mathbf{m i n})\end{array}$ & $\begin{array}{l}\text { Treadmill time } \\
(\mathbf{m i n})\end{array}$ \\
\hline Baseline & $107(37)$ & $8.2(2.8)$ & $2.1(0.5)$ & $67.1(4.5)$ & $5.2(1.6)$ \\
L fermentum & $101(42)$ & $7.6(3.2)$ & $2.4(0.6)$ & $69.5(3.8)$ & $5.6(1.4)$ \\
Placebo & $99(46)$ & $7.5(3.4)$ & $2.4(0.5)$ & $68.0(4.6)$ & $5.3(1.6)$ \\
p Value & 0.96 & 0.98 & 0.58 & 0.19 & 0.84
\end{tabular}

Results expressed as the mean (SD). Intensity of the training scored 1-5 where 1 is the least and 5 the maximum. All reported $p$ values are based on comparisons between probiotic and placebo treatments.

$12.4 \%$, respectively. For the IL12 ELISA the interassay variability was $8.4 \%$ and $9.7 \%$, respectively. Similarly, the interassay variability for IFN $\gamma$ was $3.9 \%$ and $11.9 \%$, respectively.

\section{Statistical analysis}

Simple descriptive statistics are shown as mean (betweensubject SD). Preliminary power analysis indicated that 16 subjects were required in a crossover design assuming a typical error of $12 \%$ for IFN $\gamma$, a reference change of $50 \mathrm{pg} / \mathrm{ml}$ and a type 2 error rate of $20 \%$. Mean effects of probiotic and placebo treatments and their $90 \%$ confidence limits were estimated with a spreadsheet via the unequal-variances $t$ statistic computed for change scores between pre- and post-tests of the two groups. ${ }^{22}{ }^{23}$ Each subject's change score was expressed as a percentage of baseline score by analysis of log-transformed values, in order to reduce bias arising from non-uniformity of error. Magnitudes of effect were classified against the following criteria: 0.00-0.19 trivial, 0.20-0.59 small, 0.60-1.19 moderate, 1.20-1.99 large and $>2.00$ very large. An unclear effect was defined where the confidence limits of the effect spanned the range of substantially positive to substantially negative values. Comparison of the frequency of illness measures recorded during probiotic and placebo treatments was conducted using $\chi^{2}$ analysis, and illness severity scores were compared using a Mann-Whitney $U$ test for non-parametric measures. Non-parametric statistical analyses were conducted with Statistica analytical software, version 6.0 (StatSoft Inc, OK, USA).

\section{RESULTS}

\section{Patterns of training and performance}

Table 1 shows the mean (SD) training mileage $(\mathrm{km} / \mathrm{wk})$, duration (h/wk) and intensity (scored $1-5)$. The mean training mileage was similar for both treatment groups at $\sim 100 \mathrm{~km} / \mathrm{wk}$. Similarly, there were no significant differences in training duration or intensity between treatments across the duration of the study (table 1$)$. The $\mathrm{VO}_{2 \max }(\mathrm{ml} / \mathrm{kg} / \mathrm{min})$ and maximum treadmill performance time ( $\mathrm{min}$ ) averaged for both treatments are also shown in Table 1 . The mean $\mathrm{VO}_{2 \max }$ and maximum

Table 2 Differences in the number, duration and intensity of symptoms of common upper respiratory tract infection (URTI) and lower respiratory illnesses (LRI) in highly trained distance runners between probiotic and placebo treatments

\begin{tabular}{lllc}
\hline IIIness (URTI and LRI) & L fermentum & Placebo & p Value \\
\hline Episodes (n) & 4 & 9 & 0.24 \\
Subjects reporting (n) & 3 & 7 & 0.27 \\
Symptom days (days) & 30 & 72 & $<0.001$ \\
Mean episode severity & 1.0 & 1.7 & 0.06 \\
(scored on a 1-3 scale) & & &
\end{tabular}

Severity was rated on a 1-3 Likert scale where $1=$ mild, $2=$ moderate and $3=$ severe symptoms. treadmill performance time were not substantially different between subjects receiving $L$ fermentum and placebo treatments.

Five subjects $(25 \%)$ were free of illness over the course of the study. Symptoms of URTI and LRI accounted for $58 \%$ of all reported illness episodes in the other 15 subjects. Other illness symptoms were verified as influenza, gastrointestinal illness or a skin condition. Half as many episodes of URTI and LRI symptoms were reported during the $L$ fermentum VRI-003 treatment compared with placebo (table 2); however, the mean episode duration did not differ between probiotic (7.5 days) and placebo (8 days) treatments. Similarly, fewer subjects reported respiratory symptoms during the $L$ fermentum VRI-003 treatment. The number of total illness days during $L$ fermentum VRI003 treatment $(n=30$ days) was significantly lower $(p<0.001)$ than during placebo treatment ( $\mathrm{n}=72$ days). The mean severity of respiratory illness occurring during $L$ fermentum treatment was marginally lower than that recorded during placebo treatment.

\section{Patterns of mucosal immunity}

$L$ fermentum and placebo treatments had no substantial effects on salivary IgA and salivary IgA1 concentrations (table 3 ). There was a modest increase in $\operatorname{IgA}$ after $L$ fermentum treatment and a trivial change after placebo treatment. Given the large variability in the within-subject changes with the experimental treatments the overall effect of $L$ fermentum treatment on salivary IgA was unclear. L fermentum treatment elicited a modest increase in salivary IgA1 with a trivial change after placebo. The overall effect of $L$ fermentum on salivary IgA1 was a small $26 \%$ increase compared with placebo, although this difference failed to reach statistical significance.

\section{Patterns of systemic immunity}

Table 4 shows the effects of $L$ fermentum and placebo treatments on whole-blood culture cytokine secretions. We report the baseline values at the start of each treatment, the percentage (\%) change in cytokine concentration after 28 days of supplementation, the percentage difference in the change in mean cytokine concentrations, and the magnitude (effect size) of this difference. Large between-subject variability (estimated as the coefficient of variation) was evident in the concentrations of all three cytokines under investigation (at baseline IFN $\gamma$ 285\%, IL4 $112 \%$ and IL12 118\%). There was only a trivial difference in the mean change in IL4 concentration after the experimental treatments. After $L$ fermentum treatment there was a modest increase in IFN $\gamma$, but a modest decrease after placebo treatment. The overall effect was a twofold greater increase in IFN $\gamma$ for the $L$ fermentum treatment: essentially this indicates that $L$ fermentum was effective in attenuating the reduction in IFN $\gamma$ observed in the placebo condition. Similarly, L fermentum showed some potential for attenuating reductions in IL12 with a small $36 \%$ difference in the effect of the experimental treatments. 
Table 3 Mucosal immune concentrations $(\lg A$ and $\lg A 1)$ measured in saliva at baseline and after each of the experimental treatments

\begin{tabular}{|c|c|c|c|c|c|c|}
\hline Measure & Treatment & $\begin{array}{l}\text { Baseline } \\
\text { (mg/ml) } \\
\text { Mean (SD) }\end{array}$ & $\begin{array}{l}\text { Percentage } \\
\text { change } \\
( \pm 90 \% \mathrm{CL})\end{array}$ & $\begin{array}{l}\text { Difference in } \\
\text { mean } \% \text { change } \\
( \pm 90 \% \text { CL })\end{array}$ & $\begin{array}{l}\text { Effect size } \\
\text { (descriptor) }\end{array}$ & p Value \\
\hline \multirow[t]{2}{*}{$\lg A$} & L fermentum & $56.0(35.4)$ & $29.0 \pm 80.7$ & $39.5 \pm 61.8$ & 0.59 , unclear & 0.24 \\
\hline & Placebo & $69.2(44.7)$ & $-7.5 \pm 58.9$ & & & \\
\hline \multirow[t]{2}{*}{$\lg A 1$} & $L$ fermentum & $94.5(63.4)$ & $21.3 \pm 67.0$ & $25.9 \pm 34.3$ & 0.53, small & 0.19 \\
\hline & Placebo & $92.7(34.4)$ & $-3.6 \pm 64.6$ & & & \\
\hline
\end{tabular}

The magnitude of the difference in the change in means is shown.

$\mathrm{CL}$, confidence limits.

\section{DISCUSSION}

The most important finding of this study was a significant reduction in the number of days of respiratory illness symptoms, and a trend towards a lower severity of illness, during $L$ fermentum VRI-003 treatment compared with placebo. These positive clinical consequences provide evidence for the beneficial effects of daily prophylactic intervention with $L$ fermentum in a cohort of highly trained distance runners. An improvement in resistance to common illnesses constitutes an important benefit to elite athletes undertaking high-level training in preparation for national and international competitions. In combination with the recent report that $L$ rhamonasus supplementation reduced the duration of gastrointestinal symptoms in marathon runners in the 2 weeks after the race, ${ }^{13}$ our findings point to the potential benefits of this form of nutritional intervention.

The beneficial effect of probiotic administration on the incidence of respiratory illness is possibly linked to an enhancement of systemic immunity. We observed that the IFN $\gamma$ (Th1 secreted) response was moderately higher with the probiotic treatment than with placebo. Increased IFN $\gamma$ concentration implies an increase in cell-mediated activities. ${ }^{19}{ }^{24}$ Our observation is therefore consistent with the theory that $L$ fermentum induces a Th1 shift, although this assertion awaits experimental confirmation. Gleeson and Pyne reported a substantial correlation between a decrease in salivary IgA (particularly IgA1) during training, and an increase in number of respiratory illnesses in elite athletes. ${ }^{1}$ The subclass IgA2, induced by carbohydrates and lipid-containing antigens, also offers promise as a potential marker of mucosal immune disturbance. On this basis measurement of IgA subclasses, and their ratio, may have a predictive value for infection risk in elite athletes. We observed higher IgA1 in athletes taking the probiotic, but these differences were modest and influenced by the substantial within- and between-subject variation. Future studies are required to confirm these preliminary findings.
The possibility that probiotic administration can induce a protective immune response in the respiratory tract is consistent with the findings of Gluck and Gebbers. ${ }^{25}$ These investigators showed that oral ingestion of a probiotic beverage containing a mixture of Lactobacillus GG, Streptococcus thermophilus, Bifidobacterium and Lactobacillus acidophilus elicited a substantial 19\% reduction in the occurrence of nasal pathogenic bacteria. The probiotic effects were primarily exerted on Grampositive bacteria, which decreased significantly in number, pointing to links between lymphoid tissue in the gut and the upper respiratory tract. This finding is consistent with the concept of immune cell trafficking from one mucosal site to another in the body. Stimulation at one site, such as in the intestine, can result in effects detectable at another site, such as the respiratory tract. ${ }^{25}$ Similarly, administration of Lactobacillus GG to children in day care centres decreased the incidence of respiratory tract infections. ${ }^{11}$

After colonisation of the gastrointestinal tract, the mechanisms by which probiotic bacteria mediate enhanced immunity remain unclear, with both humoral and cellular mechanisms under investigation. ${ }^{10}$ While it is not possible to draw definitive conclusions on the mechanisms by which probiotic bacteria enhance immune function from the findings of the current study, several of the trends seen are consistent with other published reports. Further investigations are required to determine whether the beneficial effects of probiotic administration can translate to both the general public and specialised populations like elite athletes, clinical conditions, immunocompromised subjects, and military and industrial settings. Since different strains of $L$ fermentum have different immunostimulating ability, ${ }^{14}$ the present findings cannot be directly extrapolated to other strains of $L$ fermentum without further testing of each strain.

We conclude that oral administration of $L$ fermentum strain VRI-003 reduced the number of days during which athletes undergoing intensive training had respiratory illnesses, possibly

Table 4 Changes in whole-blood cytokine concentrations between $L$ fermentum and placebo treatments

\begin{tabular}{|c|c|c|c|c|c|c|}
\hline Measure & Treatment & $\begin{array}{l}\text { Baseline } \\
\text { (pg/ml) } \\
\text { Mean (SD) }\end{array}$ & $\begin{array}{l}\text { Percentage } \\
\text { change } \\
( \pm 90 \% \text { CL) }\end{array}$ & $\begin{array}{l}\text { Difference in } \\
\text { mean \% change } \\
( \pm 90 \% \mathrm{CL})\end{array}$ & $\begin{array}{l}\text { Effect size } \\
\text { (descriptor) }\end{array}$ & p Value \\
\hline \multirow[t]{2}{*}{$\mathrm{IFN} \gamma$} & $L$ fermentum & $310(373)$ & $118 \pm 342$ & $199 \pm 171$ & 0.81 , moderate & 0.07 \\
\hline & Placebo & $524(521)$ & $-27 \pm 303$ & & & \\
\hline \multirow[t]{2}{*}{ IL4 } & $L$ fermentum & $54.1(39.3)$ & $-12 \pm 174$ & $0.9 \pm 70.9$ & 0.01 , trivial & 0.98 \\
\hline & Placebo & $46.7(40.9)$ & $-12 \pm 247$ & & & \\
\hline \multirow[t]{2}{*}{ IL12 } & $L$ fermentum & $182(246)$ & $-3 \pm 127$ & $36.2 \pm 38.7$ & 0.40 , small & 0.12 \\
\hline & Placebo & $168(129)$ & $-29 \pm 87$ & & & \\
\hline
\end{tabular}

The magnitude of the difference in the change in means is shown.

$\mathrm{CL}$, confidence limits. 


\section{What is known about this topic}

- Although there are hundreds of studies on probiotic administration in infants and children there is little published evidence on this practice in an athletic population.

- The potential clinical benefits of probiotic supplementation have been recognised in other populations.

- Immune stimulation is suggested as a mechanism contributing to positive clinical outcomes associated with probiotic use.

\section{What this study adds}

- These findings highlight the potential clinical and immunological benefits of probiotic administration in a group of healthy well-trained distance runners.

- The immunological benefits appear to be mediated through enhanced T-lymphocyte function.

- Probiotic supplementation may offer increased protection against viral infection in athletes.

by stimulation of a protective immune response. The finding of clinical benefits with probiotic supplementation in a small cohort of physically fit subjects, provides the basis for further studies examining the beneficial effects of $L$ fermentum VRI-003 administration on immune function in both the general community and specific populations.

Acknowledgements We thank Dr Peter French and Dr Trish Conway of Probiomics Ltd for their intellectual input, contributions and assistance throughout the project. We also thank Dr David Abraham, Department of Sports Medicine, and Graeme Allbon, Department of Physiology, Australian Institute for Sport for their clinical and laboratory support. We acknowledge the assistance of Professor Maree Gleeson, University of Newcastle and Dr Margaret Dunkley, VRI BioMedical Ltd, for their comments on the experimental design.

Funding The study was funded by Probiomics Ltd (formerly VRI BioMedical Ltd) and by an AusIndustry R\&D Start Grant awarded to VRI BioMedical. AJC was funded by VRI Biomedical Ltd as the clinical trials coordinator for this project.

Competing interests None.

Ethics approval By the Ethics Committee of the Australian Institute of Sport in accordance with the Code of Ethics of the World Medical Association (Declaration of Helsinki).

\section{REFERENCES}

1. Gleeson M, Pyne DB. Effects of exercise on the immune system: exercise effects on mucosal immunity. Immunol Cell Biol 2000; 78:536-44.

2. Gleeson M. Mucosal immune responses and risk of respiratory illness in elite athletes. Exer Immunol Rev 2000;6:5-42.

3. McDowell SL, Hughes RA, Hughes RJ. The effect of exhaustive exercise on salivary immunoglobulin A. J Sports Med Phys Fitness 1992;32:412-5.

4. Mackinnon LT, Ginn E, Seymour GJ. Decreased salivary immunoglobulin A secretion rate after intense interval exercise in elite kayakers. Eur J Appl Physiol 1993;67:1804.

5. Gleeson M, Hall ST, McDonald WA, et al. Salivary IgA subclasses and infection risk in elite swimmers. Med Sci Sports Exer 1999;17:351-5.

6. Gleeson M, Francis JL, Lugg DJ. One year in Antarctica: mucosal immunity at three Australian stations. Immunol Cell Biol 2000; 78:616-22.

7. Gorbach SL. Probiotics and gastrointestinal health. Am J Gastroenterol 2000;95(Suppl):S2-4.

8. Vanderhoof JA. Probiotics: future directions. Am J Clin Nutr 2001;73:1152-5S

9. Vrese MD, Schrezenmeir J. Probiotics and non-intestinal infectious conditions. Br J Nutr 2002:88(Suppl 1):S59-66.

10. Gill HS. Stimulation of the immune system by lactic cultures. Int Diary $J$ 1998;8:535-4.

11. Hatakka K, Savilahti E, Ponka A, et al. Effect of long term consumption of probiotic milk on infections in children attending day care centres: double blind, randomised trial. BMJ 2001;322:1327-32.

12. Clancy RL, Gleeson M, Cox A. Reversal in fatigued athletes of a defect in interferon $\gamma$ secretion after administration of Lactobacillus acidophilus. Br J Sports Med 2006; $40: 351-4$

13. Kekkonen RA, Vasankari TJ, Vuorimaa T, et al. The effect of probiotics on respiratory infections and gastrointestinal symptoms during training in marathon runners. Int J Sport Nutr Exer Metab 2007;17:352-63.

14. Plant LJ, Conway PL. Adjuvant properties and colonization potential of adhering and non-adhering Lactobacillus spp. following oral administration to mice. FEMS Immunol Med Microbiol 2002;34:105-111.

15. Gibson SAW, Conway PL. Recovery of a probiotic organism from human faeces after oral dosing. In: Gibson SAW, ed. Human health: the contribution of microorganisms. Berlin: Springer, 1994. (Springer Series in Applied Biology.)

16. Plant L, Conway PL. Association of Lactobacillus spp with Peyers patches in mice. Clin Diag Immunol 2002;8:320-4.

17. Klijn N, Weerkamp AH, de Vos WM. Genetic marking of Lactococcus lactis shows its survival in the human gastrointestinal tract. Appl Environ Microbiol 1995;61:2771-4.

18. Matusuzaki T, Chin J. Modulating immune responses with probiotic bacteria Immunol Cell Biol 2000;78:67-73.

19. Erickson KL, Hubbard NE. Probiotic immunomodulation in health and disease. J Nutr 2000;130:403-9S.

20. Farah CS, Elahi S, Pang G. T cells augment monocyte and neutrophil function in host resistance against oropharyngeal candidiasis. Infect Immunol 2001;69:6110-8.

21. Ren Z, Pang G, Lee R. The circulating T cell response to Helicobacter pylori infection in chronic gastritis. Helicobacter 2000;5:135-41.

22. Batterham AM, Hopkins WG. A decision tree for controlled trials. Sportscience 2005:9:33-9

23. Batterham AM, Hopkins WG. Making meaningful inferences about magnitudes Int J Sports Physiol Perform 2006;1:50-7.

24. Steensberg $\mathbf{A}$, Toft AD, Bruunsgaard H. Strenuous exercise decreases the percentage of type $1 \mathrm{~T}$ cells in the circulation. J Appl Physiol 2001:91:1708-12.

25. Gluck G, Gebbers J0. Ingested probiotics reduce nasal colonization with pathogenic bacteria. Am J Clin Nutr 2003;77:517-20. 